\title{
Pinning down QCD-matter shear viscosity in ultrarelativistic heavy-ion collisions via EbyE fluctuations using pQCD + saturation + hydrodynamics
}

\author{
K. J. Eskola ${ }^{\natural}$ ! H. Niemi ${ }^{b}$ and R. Paatelainen ${ }^{c}$ \\ ${ }^{a}$ University of Jyvaskyla, Department of Physics, P.O. Box 35, FI-40014 University of Jyvaskyla, Finland \\ ${ }^{b}$ Institut für Theoretische Physik, J. W. Goethe-Universität, Max-von-Laue-Str. 1, D-60438 Frankfurt am Main, Germany \\ ${ }^{c}$ Departamento de Fisica de Particulas, Universidade de Santiago de Compostela, E-15782 Santiago de Compostela, Galicia, Spain
}

\begin{abstract}
We introduce an event-by-event pQCD + saturation + hydro ("EKRT") framework for high-energy heavy-ion collisions, where we compute the produced fluctuating QCD-matter energy densities from next-to-leading order (NLO) perturbative QCD (pQCD) using saturation to control soft particle production, and describe the space-time evolution of the QCD matter with viscous hydrodynamics, event by event (EbyE). We compare the computed centrality dependence of hadronic multiplicities, $p_{T}$ spectra and flow coefficients $v_{n}$ against LHC and RHIC data. We compare also the computed EbyE probability distributions of relative fluctuations of $v_{n}$, as well as correlations of 2 and 3 eventplane angles, with LHC data. Our systematic multi-energy and -observable analysis not only tests the initial state calculation and applicability of hydrodynamics, but also makes it possible to constrain the temperature dependence of the shear viscosity-to-entropy ratio, $\eta / s(T)$, of QCD matter in its different phases. Remarkably, we can describe all these different flow observables and correlations consistently with $\eta / s(T)$ that is independent of the collision energy.
\end{abstract}

\section{Introduction}

The basic idea in the new NLO-improved perturbative QCD + saturation + viscous hydro event-by-event EKRT framework [1] presented in this talk, is as follows: Using collinear factorization and NLO pQCD, we first compute the production of transverse energy $\left(E_{T}\right)$ carried by partons of $p_{T} \sim$ few $\mathrm{GeV}$ (minijets) into the central region in $A+A$ collisions. The conjecture of gluon saturation, implemented as in the NLO-extension [2] of the original EKRT paper [3], then allows us to compute QCD-matter initial conditions, EbyE, for viscous hydrodynamics with which we describe the space-time evolution of the produced QCD matter. Comparing with a multitude of experimental RHIC and LHC data for low- $p_{T}$ observables, we aim at $(i)$ pinning down the $\eta / s(T)$ ratio, (ii) testing our initial state calculation, and (iii) studying the applicability region of viscous hydrodynamics.

\section{Minijet $E_{T}$ production from NLO pQCD}

Using collinear factorization, NLO minijet $E_{T}$ production per transverse area into a rapidity interval $\Delta y$ in high-energy $A+A$ collisions of impact parameter $\mathbf{b}$ can be computed as

$$
\frac{\mathrm{d} E_{T}}{\mathrm{~d}^{2} \mathbf{r}}=T_{A}(\mathbf{r}+\mathbf{b} / 2) T_{A}(\mathbf{r}-\mathbf{b} / 2) \sigma\left\langle E_{T}\right\rangle,
$$

where the collision geometry is given by the the standard nuclear thickness functions $T_{A}$. The minijet part [4], which in NLO [5, 2, 1] can be written as

$$
\sigma\left\langle E_{T}\right\rangle=\sum_{n=2}^{3} \frac{1}{n !} \int \mathrm{d}[\mathrm{PS}]_{n} \frac{\mathrm{d} \sigma^{2 \rightarrow n}}{\mathrm{~d}[\mathrm{PS}]_{n}} \tilde{\mathcal{S}}_{n},
$$

contains phase-space integrated $2 \rightarrow 2$ and $2 \rightarrow 3$ differential $\mathcal{O}\left(\alpha_{s}^{3}\right)$ partonic cross sections $\mathrm{d} \sigma^{2 \rightarrow n} / \mathrm{d}[\mathrm{PS}]_{n}$, obtained in terms of UV-renormalized squared matrix elements [6, 7], and CTEQ6M [8] parton distribution functions with

\footnotetext{
${ }^{1}$ Speaker at the Hard Probes 2015, Montreal, Canada, June 2015.
} 
EPS09s spatially dependent nuclear effects [9]. The measurement functions,

$$
\tilde{\mathcal{S}}_{n}=\left(\sum_{i=1}^{n} \theta\left(y_{i} \in \Delta y\right) p_{T i}\right) \times \theta\left(\sum_{i=1}^{n} p_{T i} \geq 2 p_{0}\right) \times \theta\left(\sum_{i=1}^{n} \theta\left(y_{i} \in \Delta y\right) p_{T i} \geq \beta p_{0}\right),
$$

contain step functions $\theta$ and are analogous to those in jet production [10]. They define the 1) minijet $E_{T}$ as the sum of the minijet $p_{T}$ 's in $\Delta y$;2) $p_{T}$ cut-off scale $p_{0} \gg \Lambda_{\mathrm{QCD}}$ above which we do the minijet calculation, and 3) minimum $E_{T}$ that we might require in the interval $\Delta y$. Such $\tilde{\mathcal{S}}_{n}$ ensure a well-defined NLO calculation safe from collinear and infra-red singularities. With these ingredients, the minijet $E_{T}$ calculation is field-theoretically rigorous for fixed $p_{0}$ and $\beta \in[0,1]$. As the $E_{T}$ here is not a direct observable, we must leave the parameter $\beta$ to be fixed from experimental data.

\section{Saturation in NLO minijet production}

The parameter $p_{0}$ controlling minijet production is here fixed based on a gluon saturation conjecture. As argued in [2], the $E_{T}$ production is expected to cease (saturate) when $3 \rightarrow 2$ and higher order processes become of the same magnitude as the basic $2 \rightarrow 2$ ones, $\frac{\mathrm{d} E_{T}}{\mathrm{~d}^{2} \mathbf{r d} y}(2 \rightarrow 2) \sim \frac{\mathrm{d} E_{T}}{\mathrm{~d}^{2} \mathbf{r d} y}(3 \rightarrow 2)$. This leads to a saturation criterion analogous to that in the original EKRT-model [3] but now for an IR/CL-safe $E_{T}$,

$$
\frac{\mathrm{d} E_{T}}{\mathrm{~d}^{2} \mathbf{r}}\left(p_{0}, \sqrt{s_{N N}}, A, \Delta y, \mathbf{r}, \mathbf{b} ; \beta\right)=\frac{K_{\mathrm{sat}}}{\pi} p_{0}^{3} \Delta y,
$$

from which $p_{0}=p_{\text {sat }}\left(\sqrt{s_{N N}}, A, \Delta y, \mathbf{r}, \mathbf{b} ; \beta, K_{\text {sat }}\right)$ is solved locally in $\mathbf{r}$ and for fixed $\beta, K_{\text {sat }}$. The "packing factor", proportionality constant $K_{\text {sat }}$, is to be fixed from the data. The key observation [11, 12] enabling the EbyE framework here, is that $p_{\text {sat }}$ scales essentially as $\left[T_{A} T_{A}\right]^{n}$, so that we can parametrize $p_{\text {sat }}\left(T_{A} T_{A}\right)$ vs. $\beta, K_{\text {sat }}$. The parametrization, intended also for public use, can be found in [1]. The minimum $p_{\text {sat }}$ we allow here is $1 \mathrm{GeV}$.

\section{NLO EKRT EbyE framework}

For the EbyE set-up, we first sample the nucleon positions in the colliding nuclei from the standard two-parameter Woods-Saxon density. Around each nucleon, we then set a gluon transverse density, $T_{n}(r)=\left(2 \pi \sigma^{2}\right)^{-1} \exp \left(-r^{2} /\left(2 \sigma^{2}\right)\right)$, whose width parameter, $\sigma=0.43 \mathrm{fm}$ is obtained from the exclusive electroproduction data of $J / \psi$ at HERA/ZEUS [13]. After this the thickness functions $T_{A}(x, y)$ of the nuclei can be computed, and $p_{\text {sat }}\left(T_{A} T_{A}\right)$ obtained for fixed $\beta, K_{\text {sat }}$ on the basis of the NLO pQCD+saturation calculation described above. From $p_{\text {sat }}$, we then extract the local formation time, $\tau_{s}(\mathbf{r})=1 / p_{\text {sat }}(\mathbf{r})$, and energy density

$$
e\left(\mathbf{r}, \tau_{\mathrm{s}}(\mathbf{r})\right)=\frac{\mathrm{d} E_{T}}{\mathrm{~d}^{2} \mathbf{r}} \frac{1}{\tau_{\mathrm{s}}(\mathbf{r}) \Delta y}=\frac{K_{\mathrm{sat}}}{\pi}\left[p_{\mathrm{sat}}(\mathbf{r})\right]^{4}
$$

of the minijet plasma.

"Pre-thermal" evolution from $\tau_{s}(\mathbf{r})$ to $\tau_{0}=1 / p_{\mathrm{sat}}^{\min }=0.2 \mathrm{fm}$ is here done simply with $1 \mathrm{D}$ Bjorken hydro (also free streaming would work [11]). At the edges of the collision system, below $p_{\mathrm{sat}}^{\min }=1 \mathrm{GeV}$, we connect the $e$-profile smoothly to a binary profile (see [1]).

With such EbyE initial conditions, we then run $2+1 \mathrm{D}$ viscous hydrodynamics, EbyE. Our hydro setup is the one used in [14, 15, 16, 11], i.e. 2nd-order dissipative relativistic hydro with transient fluid-dynamics equation of motion for the shear-stress tensor $\pi^{\mu \nu}$ [17, 18, 19] (see again [1]). We neglect heat conductivity and bulk viscosity and study the effects of shear viscosity only. We explore the possible temperature dependence of $\eta / s(T)$ with the parametrizations shown in Fig. 1 .

As the QCD-matter equation of state, we employ the $s 95 p$-PCE-v1 parametrization [20] with chemical decoupling at a rather high temperature of $175 \mathrm{MeV}$, and kinetic freeze-out at $100 \mathrm{MeV}$. Resonance decays after the freeze-out are included. The initial $\pi^{\mu \nu}\left(\tau_{0}\right)$ and transverse flow $v_{T}\left(\tau_{0}\right)$ are set to zero. In the non-equilibrium particle distributions on the freeze-out surface, we assume, as usual, that the relative deviations from the equilibrium distributions are proportional to $p_{\mu} p_{\nu} \pi^{\mu \nu}$ for each particle species. 


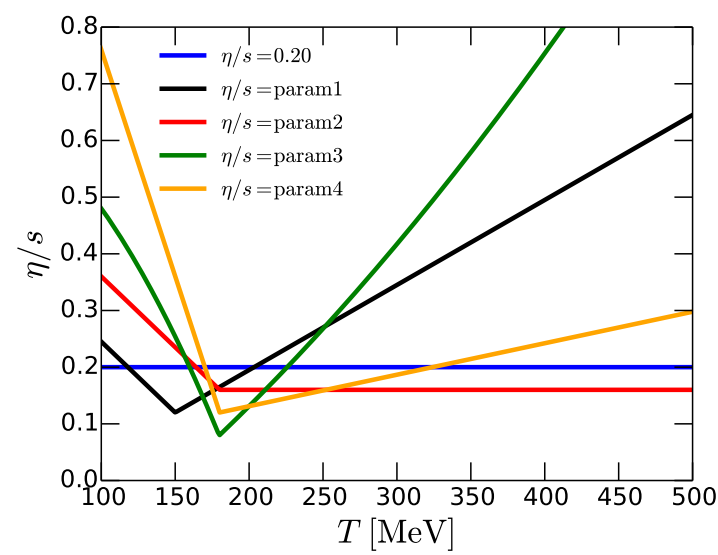

Figure 1: The tested temperature dependences of $\eta / s$. From [1].

\section{Comparison with LHC and RHIC data}

Let us then consider selected results from the extensive multi-energy and multi-observable analysis of Ref. [1]. Figure 2 shows the centrality dependence of the computed charged hadron multiplicity $d N_{c h} / d \eta$ and their flow coefficients $v_{n}\{2\}$ from the 2-particle cumulants at the LHC and $v_{2,3}\{2\}, v_{4}\{3\}$ at RHIC, and comparison with the data. For details, definitions of these quantities, and $p_{T}$ spectra, see [1]. For each $\eta / s(T)$ parametrization, and setting $\beta=0.8$ [11], we exploit only the centralmost LHC multiplicity datapoint to fix the normalization with $K_{\text {sat }}$. The good agreement with the data indicates that our initial states are on the average working well. All the $\eta / s(T)$ parametrizations of Fig. 1 reproduce the measured $v_{n}\{2\}$ 's at the LHC while the RHIC data favors a small $\eta / s(T)$ in the hadron gas phase.

Figure 3 shows the probability distributions of the charged hadron $\delta v_{2}=\left(v_{2}-\left\langle v_{2}\right\rangle_{\mathrm{ev}}\right) /\left\langle v_{2}\right\rangle_{\mathrm{ev}}$ and the initial spatial eccentricities $\delta \epsilon_{2}=\left(\epsilon_{2}-\left\langle\epsilon_{2}\right\rangle_{\mathrm{ev}}\right) /\left\langle\epsilon_{2}\right\rangle_{\mathrm{ev}}$ at the LHC in different centrality classes. Our EbyE framework catches remarkably well the centrality systematics of the measured $P\left(\delta v_{n}\right)$, and also proves that since $\delta \epsilon_{2}$ and $\delta v_{2}$ are nonlinearly correlated in non-central collisions, hydrodynamics (collectivity) is indeed required to reproduce the measured $P\left(\delta v_{n}\right)$. Furthermore, the $P\left(\delta v_{n}\right)$ offer direct constraints for the initial state in that they are insensitive to $\eta / s$.

Figure 4 shows examples of correlations of two and three event-plane angles, $\left\langle\cos \left(k_{1} \Psi_{1}+\cdots+n k_{n} \Psi_{n}\right)\right\rangle_{\mathrm{SP}}$ at the LHC. Especially since the $P\left(\delta v_{n}\right)$ now constrain our initial state calculation independently of $\eta / s$, these correlations give vital further constraints for the viscosity and for the validity of the EbyE viscous framework. Remarkably, the same two $\eta / s(T)$ parametrizations that explain the RHIC $v_{n}$ s (black and blue in Fig. 1) work best also at the LHC! Importantly, as the rightmost panel shows, for these cases also the $\delta f$ corrections remain conveniently small from central to semicentral collisions, suggesting that the obtained hydrodynamic results appear trustworthy up to 40-50\% centrality classes. For more data and discussion, consult again Ref. [1].

\section{Conclusions and outlook}

Performing a simultaneous LHC and RHIC multi-observable analysis, we have shown that the new NLO-improved EbyE EKRT framework explains remarkably consistently the LHC and RHIC bulk observables in URHIC. The framework has clear predictive power in cms-energy and centrality, and it is a promising tool for getting a controlled estimate of the $T$-dependence of the QCD matter shear viscosity, now that enough orthogonal data constraints start to be available. Our results favor a QCD-matter $\eta / s(T)$ which is between a constant value 0.2 and a modestly decreasing(increasing) in the hadron gas(QGP) phase. Similar magnitudes have been obtained also for constant $\eta / s$ by other groups [30, 31]. Next, we need a genuine global analysis to extract true statistical error limits to $\eta / s(T)$. Also the effects of bulk viscosity [32] should be studied. Work for the EKRT EbyE framework predictions for the forthcoming $5 \mathrm{TeV} \mathrm{Pb}+\mathrm{Pb}$ run at the $\mathrm{LHC}$ is in progress. 

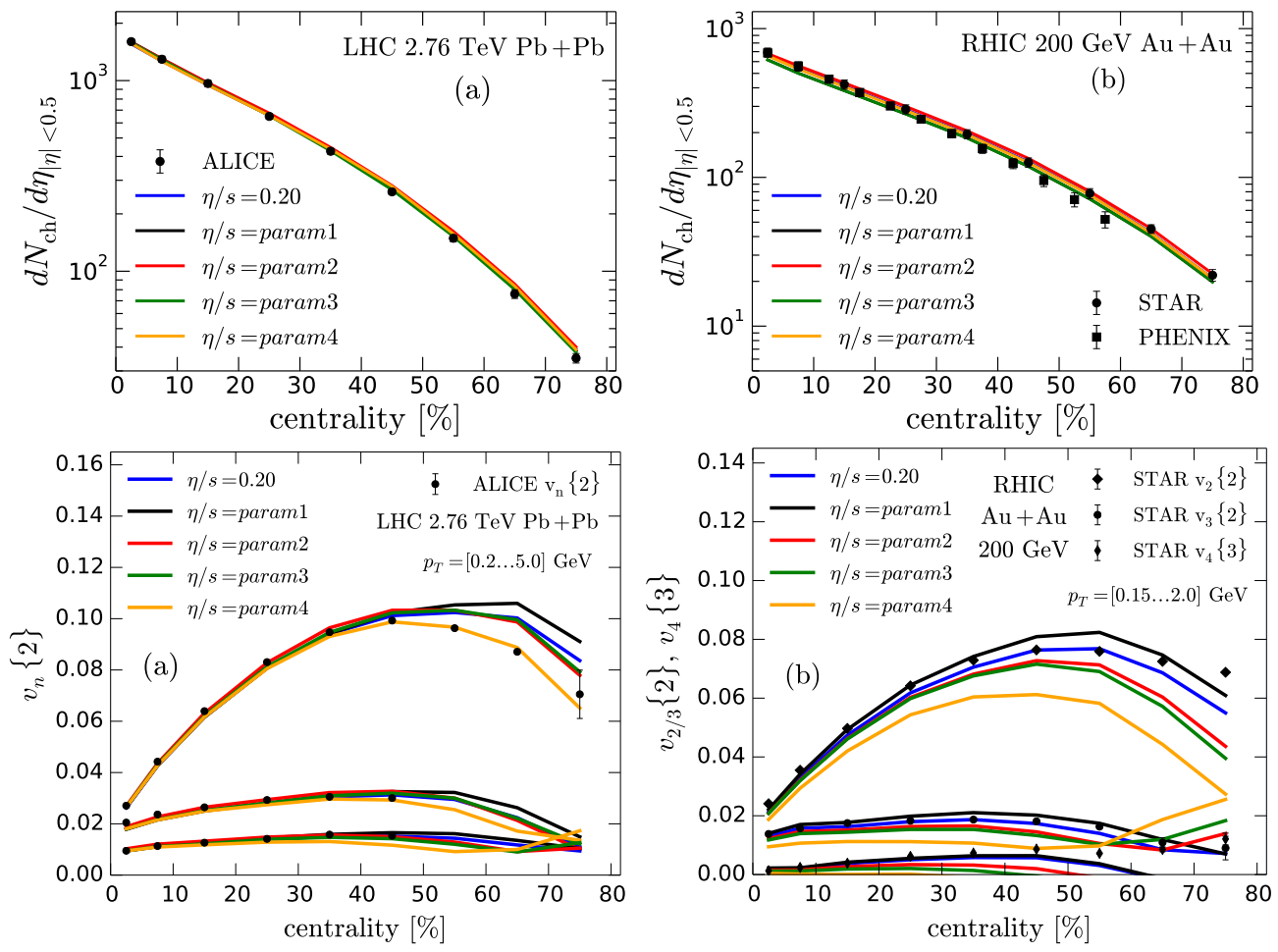

Figure 2: Upper panels: Centrality dependence of charged hadron multiplicities at the LHC (a) and RHIC (b). Lower panels: Centrality dependence of the 2-hadron cumulant flow coefficients $v_{n}\{2\}$ at the LHC (a), and $v_{2}\{2\}, v_{3}\{2\}, v_{4}\{3\}$ from the charged hadron 2- and 3-particle cumulants at RHIC (b). Experimental multiplicity data are from [21] (a) and [22, 23] (b), and $v_{n}$ data from [24] (a) and [25, 26, 27] (b). From [1].
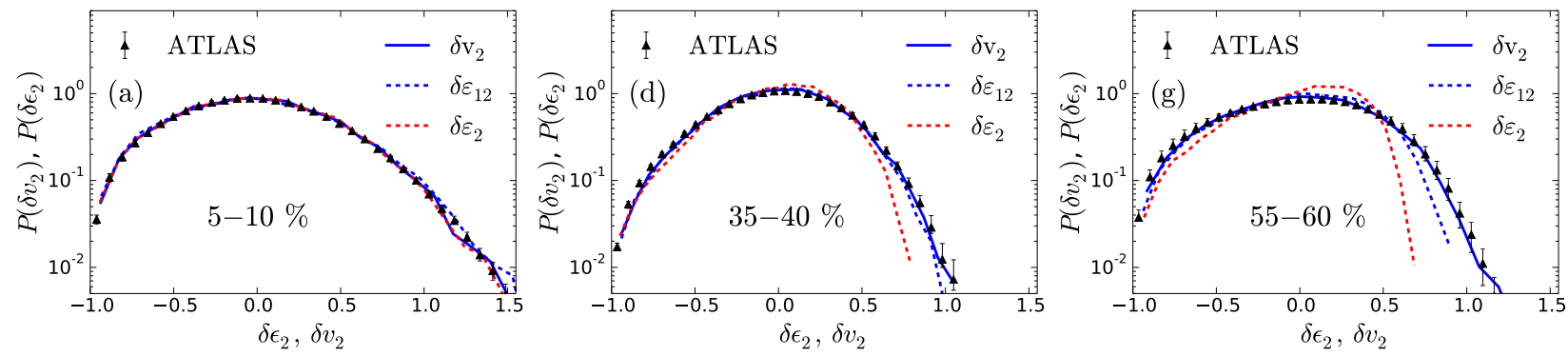

Figure 3: Probability distributions of the charged hadron $\delta v_{2}$, and of the initial $\delta \varepsilon_{2}$ (and $\delta \varepsilon_{1,2}$, see [1]) in three different centrality classes in $\sqrt{s_{N N}}=2.76 \mathrm{TeV} \mathrm{Pb}+\mathrm{Pb}$ collisions at the LHC. Figures from [1], and experimental data from ATLAS [28].
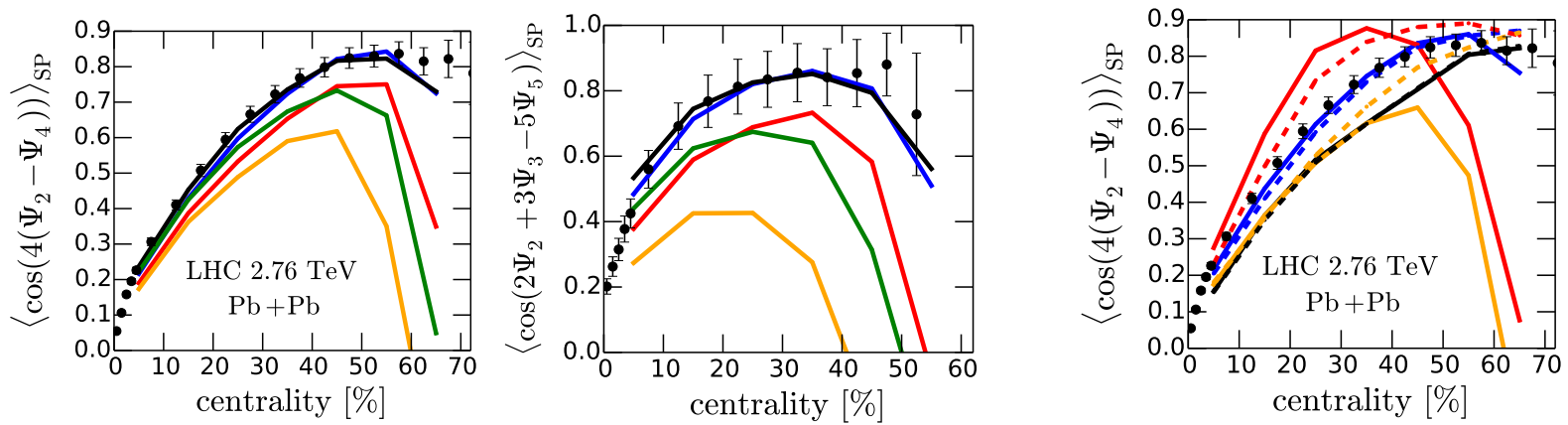

Figure 4: Examples of correlations of two (left) and three (middle) event-plane angles for charged particles at the LHC, compared with the ATLAS data [29]. Color code as in Fig. 1] Right: As the left panel but computed for $\eta / s=0.1$ (black), 0.2 (blue), 0.3 (red) and param 4 (orange) with (solid) and without (dashed) $\delta f$ corrections. From [1]. 


\section{References}

[1] H. Niemi, K. J. Eskola and R. Paatelainen, arXiv:1505.02677 [hep-ph], to appear in Phys. Rev. C

[2] R. Paatelainen, K. J. Eskola, H. Holopainen and K. Tuominen, Phys. Rev. C 87 (2013) 4, 044904

[3] K. J. Eskola, K. Kajantie, P. V. Ruuskanen and K. Tuominen, Nucl. Phys. B 570 (2000) 379

[4] K. J. Eskola, K. Kajantie and J. Lindfors, Nucl. Phys. B 323 (1989) 37

[5] K. J. Eskola and K. Tuominen, Phys. Rev. D 63 (2001) 114006

[6] R. K. Ellis and J. C. Sexton, Nucl. Phys. B 269 (1986) 445

[7] R. Paatelainen, PhD thesis arXiv:1409.3508 [hep-ph]

[8] J. Pumplin, et al., JHEP 0207 (2002) 012

[9] I. Helenius, K. J. Eskola, H. Honkanen and C. A. Salgado, JHEP 1207 (2012) 073

[10] Z. Kunszt and D. E. Soper, Phys. Rev. D 46 (1992) 192

[11] R. Paatelainen, K. J. Eskola, H. Niemi and K. Tuominen, Phys. Lett. B 731 (2014) 126

[12] K. J. Eskola, K. Kajantie and K. Tuominen, Nucl. Phys. A 700 (2002) 509

[13] S. Chekanov et al. [ZEUS Coll.], Nucl. Phys. B 695 (2004) 3

[14] H. Niemi, G. S. Denicol, P. Huovinen, E. Molnar and D. H. Rischke, Phys. Rev. Lett. 106 (2011) 212302

[15] H. Niemi, G. S. Denicol, P. Huovinen, E. Molnar and D. H. Rischke, Phys. Rev. C 86 (2012) 014909

[16] H. Niemi, G. S. Denicol, H. Holopainen and P. Huovinen, Phys. Rev. C 87 (2013) 5, 054901

[17] G. S. Denicol, T. Koide and D. H. Rischke, Phys. Rev. Lett. 105 (2010) 162501

[18] G. S. Denicol, H. Niemi, E. Molnar and D. H. Rischke, Phys. Rev. D 85 (2012) 114047 [Phys. Rev. D 91 (2015) 3, 039902]

[19] E. Molnar, H. Niemi, G. S. Denicol and D. H. Rischke, Phys. Rev. D 89 (2014) 7, 074010

[20] P. Huovinen and P. Petreczky, Nucl. Phys. A 837 (2010) 26

[21] K. Aamodt et al. [ALICE Coll.], Phys. Rev. Lett. 106 (2011) 032301

[22] B. I. Abelev et al. [STAR Coll.], Phys. Rev. C 79 (2009) 034909

[23] S. S. Adler et al. [PHENIX Coll.], Phys. Rev. C 71 (2005) 034908 [Phys. Rev. C 71 (2005) 049901]

[24] K. Aamodt et al. [ALICE Coll.], Phys. Rev. Lett. 107 (2011) 032301

[25] J. Adams et al. [STAR Coll.], Phys. Rev. C 72 (2005) 014904

[26] L. Adamczyk et al. [STAR Coll.], Phys. Rev. C 88 (2013) 1, 014904

[27] J. Adams et al. [STAR Coll.], Phys. Rev. Lett. 92 (2004) 062301

[28] G. Aad et al. [ATLAS Coll.], JHEP 1311 (2013) 183

[29] G. Aad et al. [ATLAS Coll.], Phys. Rev. C 90 (2014) 2, 024905

[30] H. Song et al., Phys. Rev. Lett. 106 (2011) 192301 [Phys. Rev. Lett. 109 (2012) 139904]

[31] C. Gale et al., Phys. Rev. Lett. 110 (2013) 1, 012302

[32] S. Ryu et al. arXiv:1502.01675 [nucl-th] 